\title{
Gouvernance verticale, corruption et clientélisme
}

Le cas de l'aide alimentaire en Inde urbaine

Frédéric Landy

\section{CpenEdition}

Journals

Édition électronique

URL : https://journals.openedition.org/ress/2811

DOI : $10.4000 /$ ress.2811

ISSN : 1663-4446

Éditeur

Librairie Droz

\section{Édition imprimée}

Date de publication : 27 novembre 2014

Pagination : 105-131

ISBN : 978-2-600-01866-1

ISSN : 0048-8046

Référence électronique

Frédéric Landy, "Gouvernance verticale, corruption et clientélisme », Revue européenne des sciences sociales [En ligne], 52-2 | 2014, mis en ligne le 01 janvier 2018, consulté le 21 septembre 2021. URL http://journals.openedition.org/ress/2811 ; DOI : https://doi.org/10.4000/ress.2811 


\title{
GOUVERNANCE VERTICALE, CORRUPTION ET CLIENTÉLISME LE CAS DE L'AIDE ALIMENTAIRE EN INDE URBAINE
}

\author{
FRÉDÉRIC LANDY \\ Université Paris Ouest-Nanterre - Mosaïques-Lavue et CEIAS \\ frederic_landy@orange.fr \\ Avec la collaboration de \\ Thomas François, Céline Hachet, Donatienne Ruby et Peeyush Sekhsaria
}

\begin{abstract}
Résumé. Les relations entre démocratie et «petite corruption» restent assez peu étudiées. Comment expliquer que l'Inde, «la plus grande démocratie du monde», connaisse un bon fonctionnement de ses institutions constitutionnelles tout en ayant un si fort niveau de corruption? Notre étude de la distribution alimentaire subventionnée montre que la corruption trouve un terreau propice dans les bases mêmes de la démocratie urbaine. D'abord sont décrites les malversations auxquelles prête l'aide distribuée par l'État dans les quartiers pauvres de Bombay (Mumbai) et de Hyderabad. À travers l'analyse de la «chaîne de corruption» nous mettons ensuite en évidence la verticalité du capital social dominant et de la gouvernance urbaine. Comment parler de «société civile» dans un tel contexte? Nous terminons en réexaminant à la lumière de l'Inde les grandes «logiques» expliquant la corruption africaine jadis explicitées par Olivier de Sardan (1999), afin de déterminer quelles sont celles qui se prêtent à une généralisation pour tous les pays du Sud voire au delà.
\end{abstract}

Mots-clés: aide alimentaire, bidonville, clientélisme, corruption, Inde, gouvernance.

\begin{abstract}
Few studies address the relationships between democracy and "little corruption". How to explain that India, "the largest democracy in the world", suffers from a high level of corruption while its constitutional institutions are working properly? This research on subsidized food distribution shows that corruption can flourish on the very bases of urban democracy. First the article highlights the embezzlement regarding state-distributed food in poor areas of Mumbai and Hyderabad. It then describes the "corruption chain" and the verticality of both social capital and urban governance is pointed out. The notion of "civil society" appears very debatable in such a context. Finally, the Indian case study reassesses the main "logics" explaining corruption in Africa that Olivier de Sardan (1999) had brought out. The goal is to check which logics are valid for all the developing countries and even beyond.
\end{abstract}

Keywords: corruption, food distribution, governance, India, patronage, slum. 
Ce matin il y a peu de monde devant le bureau de quartier de l'aide alimentaire

(Rationing Office). Nous interrogeons un intermédiaire (agent), gros homme assez bien habillé, qui attend dans la cour. Au début, c'est Io min de langue de bois. «Oui, j’amène les usagers au bon comptoir, je les aide à remplir les formulaires, je leur prends dix roupies ${ }^{1} . . . »$ Et puis soudain il s'arrête, et chuchote à mon interprète : «Vous voulez qu'on parle? Dans un restaurant tranquille? Pour i oo० Rs je vous dis tout ». On marchande à $500 \mathrm{Rs}$ (première fois que je paie ainsi une information!), et nous voilà dans un restaurant végétarien populaire. Je commande des idli, lui ne prend rien car c'est ramadan et il est musulman - pendant tout l'interview il va presser régulièrement son chapelet mécanique. Il me débite une impressionnante liste de prix et de magouilles. Par exemple, comment faire un faux livret familial d'alimentation. «Imaginez que ce soit un chrétien qui me fasse la commande. Je contacte alors un secrétaire au bureau d'un autre arrondissement, et lui demande d'ajouter le nom de mon client sur un vieux livret qu'il a gardé clandestinement et qui a appartenu à une famille chrétienne (pour le nom, cela fera plus réaliste). Deux semaines plus tard on demande le transfert de cette personne vers mon bureau, et on peut lui faire alors un livret en toute légalité, et notre Rationing Officer et moi on est couverts! Pour cette combine je prends jusqu'à I5 000, mais en général 8 ०००. Je garde 2000 et le reste va au Rationing Officer qui redistribue ».

Carnet de terrain, 25 sept. 2006, Gilbert Hill, Bombay.

La «bonne gouvernance» telle qu’elle est encouragée par les organismes internationaux implique de mettre en place des processus de libéralisation et de décentralisation, mais aussi de transparence (accountability). Tous ces éléments cohabitent-ils pour autant facilement? On peut se demander au contraire si le retrait relatif de l'État par rapport aux acteurs privés et aux collectivités locales élues, loin d’encourager la transparence, ne risque pas de développer la corruption et de multiples dysfonctionnements : la multiplication des niveaux de décision et des acteurs peut aussi représenter la multiplication des opportunités pour faire jouer les relations de pouvoir et de personnes, le népotisme et le clientélisme.

L'Inde a mis en œuvre des politiques de libéralisation et de décentralisation depuis le début des années 1990. Jamais les scandales ne semblent avoir été aussi nombreux, impliquant des sommes de plus en plus énormes². Pourtant, la relation directe entre libéralisme et corruption, avancée par les medias de gauche,

I En septembre 2006 I euro valait 56 roupies (Rs) - 80 en août 2013 .

2 Les fonctionnaires auraient reçu 10 milliards de dollars de pots-de-vin selon une estimation pour 2006, et l'économie souterraine représenterait jusqu'à 40\% du PIB. 
doit être fortement nuancée. La corruption existait bien avant les réformes, alors même que régnait l'État-providence fondé par Nehru. En outre, la «petite corruption», celle qui frappe au quotidien l'Indien moyen (loin de la «grande corruption» qui implique ministres et grosses entreprises) - ces pratiques qui parfois ne concernent que quelques centaines de roupies chacune mais dont l'addition représente un montant colossal -, apparaissent sans fortes relations avec les changements politiques. Certes, l'enseignant du collège public s'arrange parfois pour que ses élèves prennent des cours particuliers avec lui, et l'absentéisme de certains instituteurs rivalise avec celui de médecins d'hôpital préférant consulter en clinique privée: tout ceci peut être mis en relation avec une politique qui délaisse le secteur public et encourage le privé dans ce qui jusque là était considéré comme un bien public (éducation, santé, etc.). Mais le permis de conduire qu'il faut acheter, la carte d'identité pour laquelle un bakchich est demandé, le marché proposé par le policier qui vous propose d’effacer votre lourde amende contre un petit pot-de-vin, rien de tout cela ne paraît lié aux réformes en cours.

La corruption est l'utilisation à des fins privées d'une position dans le service public (on la distinguera ici des dessous de table et autres évasions fiscales impliquant uniquement des acteurs privés). Alors que la «grande corruption » existe dans tous les pays, la «petite» apparaît plus spécifique aux pays du Sud. Curieusement, la littérature scientifique est assez limitée sur ce sujet - peut-être plus par souci du politiquement correct que par les risques et les difficultés d'une telle recherche (Blundo, Olivier de Sardan, 2007). On l'explique souvent par des raisons économiques : les fonctionnaires sont mal payés, ou payés en retard, ou point payés du tout. De telles explications apparaissent peu probantes pour l'Inde, où les fonctionnaires jouissent de salaires relativement confortables (même s'ils n'ont pas suivi la hausse des cadres du privé), avec un système de retraite et de sécurité sociale que peuvent envier les 90\% d'emplois existant en Inde dans le secteur informel... Il convient de toute façon de sortir d'une vision économiste trop centrée sur l'intérêt individuel et oubliant les structures sociales qui l'enchâssent (Véron et al., 2006)³.

3 La nouvelle de Shroff (2008) décrivant les affres d'un fonctionnaire qui ne cède à la corruption, après plusieurs années, que parce qu'il doit «bien» marier et doter sa fille, illustre l'importance de la pression collective au lieu de l'appât du gain individuel. 
Comment comprendre alors «l'absence d'éthique de service public», sauf à chercher du côté des structures socio-culturelles? C'est l'objet de cet article, qui souhaite contribuer à l'étude des relations entre démocratie et corruption. Comment expliquer que «la plus grande démocratie du monde $»^{4}$ connaisse un bon fonctionnement de ses institutions constitutionnelles tout en ayant un si fort niveau de corruption? Cette dernière ne trouve-t-elle pas paradoxalement un terreau propice dans les bases mêmes de la démocratie urbaine (associations locales, forte participation électorale des populations pauvres, récente décentralisation)?

Sans doute faut-il chercher du côté du clientélisme (patronage), ce «système d'échanges interpersonnels non marchands de biens et de services échappant à tout encadrement juridique entre agent de disposant de ressources inégales » (Briquet, Sawiki, I998, p. 2). Ses trois composantes clés : «la personnalisation, la réciprocité et la dépendance » (ibid., p. 7). L’inégalité à la base du clientélisme rend cette structure éminemment verticale, reliant des personnes ou des groupes dominants à des dominés. Le clientélisme est donc souvent considéré comme un obstacle à la formation «horizontale» de classes au sens marxiste du terme, puisque la solidarité potentielle entre personnes de même rang économique ou de même travail est transpercée par des relations clientélistes entre personnes de rangs différents. Or, la société indienne apparaît très favorable au clientélisme, étant donné la permanence d’une segmentation entre communities, qu'elles soient de caste (ou de sous-caste!), de confession, d'origine régionale, de langue, etc. La structure de castes ne fonctionne pas comme un empilement de feuillets horizontaux et hiérarchisés : unissant souvent des pauvres et des riches, des puissants et des misérables, la caste est le siège de relations clientélistes. De même, des personnes de même origine géographique peuvent être solidaires quoique de niveaux socio-économiques très différents. Cette verticalité des structures sociales a été analysée d’abord dans les études de villages, où nombreuses sont les factions (Srinivas, 1976). Elle existe aussi, nous le verrons, en milieu urbain.

4 Expression par laquelle les Indiens aiment qualifier leur pays, dépassé en population seulement par la Chine. 
Le clientélisme est en général un moyen pour donner accès à des biens et services qui, sinon, seraient restés hors de portée. La corruption économique peut donner elle aussi accès, mais elle représente souvent un obstacle à ces biens et services, notamment dans le cas où ces derniers sont théoriquement publics et gratuits mais ne peuvent être délivrés que contre un pot-devin (Kumar, Landy, 2009). Le clientélisme est un facilitateur, la corruption est souvent un frein (que le clientélisme fonctionne comme un frein aux yeux de ceux qui ne font pas partie du réseau est une autre question...). On retrouve semblable ambiguïé dans un personnage commun à beaucoup de sociétés des pays du Sud, le courtier (broker appelé souvent en Inde agent, en hindi dalal). Celui-ci peut avoir deux fonctions opposées. Cet intermédiaire peut ouvrir des portes, donnant accès, comme le montre l'exergue de cet article, à des services inaccessibles autrement - et ce, souvent à travers du clientélisme. Mais il peut aussi fermer ces portes, refusant l'accès à ceux qui ne veulent ou ne peuvent payer un pot-de-vin. Brokerage peut se traduire par «médiation» tout comme par «filtrage». Cet article apportera des exemples de cette ambiguïté. Dans une première partie sont décrites les malversations auxquelles prête l'aide alimentaire distribuée par l'État dans les quartiers pauvres de Bombay (Mumbai) et de Hyderabad. En deuxième partie, la «chaîne de corruption » met en lumière la verticalité du capital social dominant et de la gouvernance urbaine. Comment parler de «société civile » dans un tel contexte? On terminera en réexaminant à la lumière de l'Inde les grandes «logiques» expliquant la corruption africaine jadis explicitées par Jean-Pierre Olivier de Sardan (1999), afin de déterminer quelles sont celles qui se prêtent à une généralisation pour tous les pays du Sud voire au delà.

\section{UN SYSTÈME DE DISTRIBUTION «PUBLIQUE » ALIMENTAIRE QUI N'EST POINT SI PUBLIC}

Notre enquête a eu lieu à Bombay et Hyderabad entre 2005 et 2007 (avec une actualisation rapide pour Bombay en 2013). Elle s'est faite dans le cadre du programme ACI Acteurs, Politiques et Gouvernance Urbaine qui cherchait à rendre compte des changements en Inde dans la gestion et l'accès aux services publics qui pourraient traduire une nouvelle gouvernance urbaine portée par la décen- 
tralisation et la libéralisation (Ruet, Tawa Lama-Rewal, 2009). À Hyderabad, 60 ménages ont fait l'objet d'enquêtes dans le quartier de Sultan Shahi, un bidonville consolidé de la vieille ville, ainsi que 53 ménages dans deux villages périurbains. À Bombay, deux bidonvilles ont été sélectionnés, Gilbert Hill à Andheri West (enquêtes sur 3 I ménages) et Antop Hill à Matunga East, ainsi que le quartier bourgeois de Parsi Colony (Matunga) (49 interviews). Des études aussi réduites par rapport à l'immensité de deux mégapoles de respectivement 8 et I 8 millions d'habitants (recensement de 20II), capitales de l'Andhra Pradesh et du Maharashtra, ne prétendent pas à la représentativité statistique. Mais leur visée avant tout qualitative permet de mettre en lumière de façon détaillée et empirique certains aspects de la gouvernance urbaine à l'indienne.

Le Public Distribution System (PDS) est un réseau par lequel transitent huile et pétrole lampant (avant tout importés) mais aussi blé, riz, sucre qui proviennent de stocks nationaux alimentés par les achats de l'État au titre du soutien des prix agricoles. La politique alimentaire a tout à la fois un volet productiviste et un volet social : d'une part elle garantit des prix stables aux agriculteurs céréaliers afin de les encourager à intensifier leur production, d'autre part elle redistribue à prix subventionnés les stocks constitués par les achats de l'État - ce qui théoriquement doit contrebalancer la tendance inflationniste du premier volet (Landy, 2006). Tout ménage indien détient en principe un livret d’alimentation (ration card) qui, sauf pour les familles aisées, donne droit à des quantités de vivres et à des prix variant en fonction du revenu du ménage. Les produits sont vendus dans une boutique de quartier spécifique (ration shop, fair price shop) où est inscrit le ménage.

L’aval du PDS est géré par les États fédérés et non par New Delhi. Les règles changent donc selon qu'on se trouve en Andhra Pradesh ou au Maharashtra: prix, quantités, seuils de pauvreté, etc. Ce seuil de pauvreté détermine deux types de livrets, pour les ménages pauvres et pour les «non-pauvres» (d’autres catégories existent pour les plus riches au Maharashtra, et dans les deux États pour les ménages les plus misérables). La première difficulté de la véritable «course d'obstacles» (Landy et al., 20I3) à laquelle font face les ménages est d'obtenir un livret - et si possible, pour les familles sous le seuil de pauvreté, un livret de «pauvre» afin d’obtenir les prix et quantités les plus intéressants. Or, dans 
les villages périurbains de Hyderabad, un quart des ménages enquêtés n'avait aucun livret, $56 \%$ de ceux qui en disposaient avaient un nombre de personnes mentionnées dessus inférieur à la taille de la famille, tandis que $47 \%$ des livrets pour «pauvres» appartenaient à des ménages au dessus du seuil de pauvreté. Dans la vieille ville de Hyderabad, I5\% de notre échantillon n’avait aucun livret. La situation était pire à Bombay, avec un tiers des enquêtés de Gilbert Hill dans ce cas. À Antop Hill, toutes les familles habitant dans le bidonville consolidé avaient un livret (mais pour «non-pauvres »!) tandis que moins de $5 \%$ de celles vivant dans le quartier de huttes voisin, plus récent, en disposaient.

À Bombay, pour obtenir le livret il faut passer par un intermédiaire (agent) qui empêche l'accès au bureau d'enregistrement de quartier. On doit payer des sommes allant jusqu'à I 5000 Rs, soit le revenu d'une centaine de jours de travail non qualifié, pour obtenir le précieux sésame. (Les agents laissent passer les classes moyennes et supérieures car elles ont trop d'entregent). Le PDS est donc peu «public » à deux titres : d'une part, nombre de citoyens n'y ont pas accès ; et, d’autre part, il est partiellement «privatisé» au profit de quelquesuns. L'argent est ensuite redistribué par le courtier aux différents fonctionnaires impliqués, qui peuvent ainsi garder l'anonymat (Landy et al., 20I3). Dans la vieille ville de Hyderabad, les sommes demandées sont plus faibles, servent plus à faire accélérer les choses qu’à véritablement les débloquer (speed money, tchaï panis ${ }^{5}$, et les agents sont moins systématiques; en zone périurbaine, il n’y a même rien de tout cela, nous verrons pourquoi.

Même munis du livret de la bonne couleur, les ménages subissent de nouvelles exactions à la ration shop, étant donné que le gérant triche de multiples façons (sable dans le blé, inscription dans le livret de quantités vendues supérieures à la réalité, etc.) pour pouvoir revendre sur le marché libre (il possède souvent plusieurs «vrais-faux» livrets obtenus de ménages désormais émigrés, ou d’un vieillard décédé). Lui-même peut se défendre en rappelant qu’il est rémunéré selon une marge fixée à un niveau très faible par l'État, et qu'il se trouve lui-même victime 
de «fuites» quand les sacs commandés arrivent sous-remplis : il n'empêche que sa tâche est au final fort lucrative, ce qui fait que la concurrence pour obtenir une telle charge de l'administration donne lieu à corruption et truquages ${ }^{6}$. Convenons aussi que beaucoup de ménages «trichent» également - dans une proportion qui n'est certes pas comparable: certains ont deux cartes (parents décédés ou émigrés) ; des familles qui n’ont pas droit au sucre ou au pétrole en achètent quand même. Le gérant en est souvent conscient mais laisse faire contre un pot-de-vin.

Même si le PDS est loin d'être le seul secteur corrompu de l'administration indienne, il est connu pour être un de ceux où circule le plus d’argent occulte, et ce, jusquau ministre de l'Alimentation. Pour en rester aux niveaux inférieurs, on peut se demander pourquoi certains ménages à Bombay sont prêts à de lourds sacrifices en argent et en temps pour obtenir un livret, alors que les produits sont de qualité médiocre. La réponse est multiple. D’une part, les prix sont très subventionnés pour les plus pauvres (du riz est vendu 2 Rs le kilo en Andhra Pradesh, ou gratuit pour certains ménages, alors qu’à qualité égale il vaut souvent plus de I5 Rs sur le marché libre). Même si les quantités autorisées ne dépassent pas $35 \mathrm{~kg}$ mensuels par ménage de cinq personnes, le PDS a un grand rôle à jouer dans une Inde où les dépenses alimentaires concernent encore la moitié du budget moyen familial. La terminologie employée (rationing) évoque encore le temps des pénuries et des famines, époque pourtant révolue à l'échelle nationale depuis les succès de la révolution verte. À cet enjeu national s'ajoutent des particularités locales. Dans les deux villes le PDS jouit d'une image remarquable, qui fait que somme toute il marche moins mal quailleurs: c'est à Bombay que naquit l'aide alimentaire publique, dès 1942. Et c'est en Andhra Pradesh quapparut la politique alimentaire la plus généreuse, lancée par le célèbre homme politique N. T. Rama Rao en 1983.

Mais les enjeux alimentaires ne sont pas seuls. Obtenir un livret, c'est aussi jouir par là même d’un certificat de résidence étant donné que l'adresse du ménage y est indiquée - document précieux si l'on vit en bidonville. C'est enfin jouir d'une carte d'identité dans un pays où celle-ci est encore rare - un atout en 
particulier si l'on est musulman, qui permet d'échapper au soupçon d'être un Bangladais clandestin ou un Pakistanais terroriste en puissance (Landy et al., 20I3).

Voilà sans doute pourquoi dans les villages périurbains de Hyderabad il n’y a pas d'agents: les enjeux du PDS se trouvent moindres en zone semi-rurale, étant donné qu'ils ne sont qu'alimentaires. Là, pas de «terrorisme», moins de craintes d'étrangers, et pas de bidonvilles qui risquent d'être détruits. On est loin de la situation de Bombay, où se développe un cercle vicieux sans fin: plus le livret est recherché et plus les bakchichs augmentent. Plus le livret est un objet de commerce et plus il est entouré de suspicion par l'administration, ce qui multiplie les contrôles et, partant, l'intensité de la corruption : car plus il y a d'inspections, et plus il y a d’argent qui circule. «Vous pouvez devenir citoyen de ce pays pour 3000 Rs! », nous dit un élu local du parti nationaliste hindou BJP. De fait, le pot-de-vin a une signification très différente selon le point de vue: puisqu'il faut le payer pour obtenir un livret, c'est un obstacle à l'accès au service public de l'alimentation subventionnée; mais pour cet élu et pour tous ceux qui pensent que l'immigration menace l'identité locale ou nationale, le pot-de-vin est un sésame qui donne accès à une nationalité pour le reste difficile à obtenir.

À moins d'un découplage effectif de la question alimentaire et de la question identitaire, le PDS ne pourra remplir son rôle premier, qui est l'obtention de la sécurité alimentaire. Si le livret perdait sa fonction de carte d'identité, il donnerait lieu à moins de contrôles, moins de convoitises, moins de corruption. Pour l'instant, l'association alimentation-identité a des conséquences négatives dans les deux domaines: elle limite l'accès aux vivres subventionnés, et elle crée la suspicion sur l'identité des individus puisque le livret est achetable. Créer un véritable état-civil en Inde est donc sans doute un moyen indirect mais très efficace pour lutter contre la corruption dans le PDS. L'enregistrement numérique en cours de l'ensemble de la population indienne (programme Adhar) va dans ce sens, même s'il pose d'autres problèmes : erreurs dans l'enregistrement des empreintes digitales et de l'iris, libertés des citoyens, etc., sans parler de la nécessité de vérifier l'identité, donc souvent d’exiger le livret d’alimentation! 


\section{INFORMALITÉ DES ACTEURS OFFICIELS, FORMALITÉ DES ACTEURS INFORMELS}

\section{I. LA CHAÎNE DE CORRUPTION \\ Des maillons formant système}

L'exergue de cet article témoigne d'une chaîne de corruption et de complicité existant à l'intérieur de l'administration, reliant différents niveaux de pouvoir et de compétence (Landy, 2006). Les petits fonctionnaires reversent à leurs supérieurs une bonne partie de leurs pots-de-vin, pour se maintenir à leur poste ou en obtenir un plus élevé. Mais cette chaîne a d'autres maillons plus extérieurs. Même si des données précises nous manquent, il est vraisemblable qu'on retrouve la structure triangulaire décrite jadis par Wade (1985) à propos de l'irrigation : le fonctionnaire, le parti politique et l'usager, avec une collusion (nexus) entre les deux premiers dont est victime le dernier. De fait, c'est bien l'absence d'étanchéité entre le milieu politique et l'administration qui en Inde est une source majeure de corruption (Das, 200I). Les pots-de-vin financent directement le parti, ou bien le fonctionnaire qui reverse au parti pour que celui-ci appuie sa demande de promotion dans le cadre d'un véritable «marché des postes » (Wade, 1985). Élément supplémentaire, qui complexifie le système et facilite la corruption : la présence du secteur privé. Qu'il soit l'entrepreneur en travaux publics dans le cas des programmes d’emploi rural (Véron et al., 2006) ou le gérant de ration shop, cet individu est un maillon de plus dans la «chaîne de corruption » qui s'interpose entre le citoyen et le service pourtant dû.

Cette chaîne déborde donc de la filière alimentaire, non seulement parce que le fonctionnaire peut reverser des bakchichs à l'élu local ou à la cellule du parti dominant le quartier, mais aussi parce que les agents jouent un rôle essentiel de collecte et de redistribution des pots-de-vin. Éléments «extérieurs», ils le sont parce qu'ils ne font pas officiellement partie de l'administration; ils se tiennent le plus souvent hors du bâtiment officiel. Ils ont toutefois un statut très ambigu : travaillant dans la cour bordant le bâtiment, ayant leurs entrées auprès de certains fonctionnaires, se faisant parfois même passer pour l'un d'entre eux, ils correspondent à une «sous-traitance de la corruption » à peine externalisée, à de l'outsourcing de proximité, pour ainsi dire. De même qu'en Inde de petits 
ateliers relevant du secteur informel peuvent jouxter une usine moderne pour laquelle ils fabriquent à bas coûts des composants, de même dans le cas du PDS ces agents sont utilisés par les fonctionnaires pour leur permettre d'optimiser leurs revenus illégaux sans pour autant prendre le risque d'opérer en plein jour.

C'est ce qui rend la lutte contre la corruption si difficile : elle n'est pas le fait d'un individu ou d'un niveau de la filière, mais de tout un système. La lutte par l'administration elle-même est avant tout négative, à travers une augmentation des contrôles et des interdictions. Les Rationing Inspectors inspectent davantage les usagers que les ration shops et les fonctionnaires! L'introduction de coupons puis de cartes à code barres, précoce en Andhra Pradesh, l'informatisation progressive du système pour détecter les fausses cartes, sont des actions plus «positives » mais qui n'empêchent pas fraudes et exactions.

\subsection{UNE ILLÉGALITÉ AVEC «PIGNON SUR RUE»: LES AGENTS}

Selon une des rares enquêtes un tant soit peu quantitatives qui existent sur l'Inde (Bertrand et al., 2008), quelque 60\% des titulaires du permis de conduire à Delhi l'ont acheté sans le passer; ils l'ont payé en moyenne 2,5 fois plus cher que ce que requiert le timbre... et pour les trois quarts ils ont utilisé, le plus souvent sans en avoir le choix, un courtier auprès de qui ils ont payé un pot-de-vin. Selon cette même enquête, $54 \%$ des enquêtés qui avaient un livret d'alimentation l'avaient obtenu par un agent, tout comme $47 \%$ de ceux qui détenaient un titre de propriété, I5\% des titulaires de passeport et $20 \%$ d'un compte bancaire. L’utilisation d'un tel intermédiaire est assez générale, dans les grandes villes comme à la campagne.

Ce sont parfois des femmes. Comme l'a montré Goetz (2007), il est fort douteux que les femmes soient par nature ou du fait de leur situation sociale moins sujettes à la corruption que les hommes. Dans des sociétés patriarcales comme l'Inde, les femmes dans la politique ou le secteur public ont moins de possibilités de soutirer des pots-de-vin étant donné que leur sexe leur interdit certaines interactions en dehors de leur réseau familial. En revanche, dans les milieux à dominante féminine, ce genre de restriction disparaît - à l'instar des sages-femmes en Afrique (Olivier de Sardan, 200I). 
Quand les agents féminins du PDS ont pour client une femme, elles ne sont plus contraintes par les dangers de situations intersexuées, et du coup se comportent à l'image de leurs collègues hommes.

Les bidonvilles étudiés à Bombay sont peuplés de maharashtriens, mais aussi de musulmans ourdouphones, de tamouls, etc. La mosaïque linguistique des grandes villes indiennes complique la tâche de l'administration. Dans l'organigramme de l’administration des Civil Supplies, il existe en général un clerc chargé d’aider les usagers à remplir les formulaires ; mais le fonctionnaire est rarement présent en réalité. La place est donc libre pour les agents. Contrairement aux «courtiers en développement» africains (Bierschenk et al., 2000) ou à ceux du système de microcrédit indien (Guérin, Kumar, 2007), ils apparaissent ici non comme interface entre projet et population mais comme insérés dans le fonctionnement interne du programme. En tout cas, ils sont moins courtiers (brokers), et encore moins facilitateurs ${ }^{7}$ (fixers), que filtreurs. Ce sont des courtiers dans le sens anglais du terme, des courtisans : en attente, non pas à la cour du souverain, mais dans la cour du Rationing Officer. Une autre caractéristique est qu'ils sont presque tous «protégés » par des acteurs «officiels» de la gouvernance urbaine locale: soit par les fonctionnaires de l'administration du PDS, soit par les élus, soit par les associations locales.

\subsection{LES AUTRES ACTEURS DE LA GOUVERNANCE LOCALE DU PDS}

Ils sont actifs en tant que protecteurs des agents, ou bien ils agissent directement auprès de la population (Landy et al., 2013). C'est le cas de l'administration du ministère de l'Alimentation: véritables «bureaucrates d'interface » entre État local et usagers (Olivier de Sardan, 20I4), les fonctionnaires savent manier pour la plupart à la fois les normes officielles, qui cherchent à limiter la corruption, et les «normes pratiques» (Olivier de Sardan, 200 I et 20I4) de la corruption et des passe-droits. Ces dernières font de l'État indien, au contact

7 Voir notamment les despachantes brésiliens, facilitateurs qui contre un paiement souvent modeste permettent l'accès aux services et sont une courroie essentielle du jeitinho, de la «débrouille» (voir Rosenn, 197| et Levine, 1997:<http://www.brazzil.com/blajan98.htm>).

8 Les ONG et la question de la mobilisation populaire sont traitées dans Landy et al., 2013. 
avec les agents, avec les gérants privés des ration shops et avec toutes sortes de transactions officielles ou occultes, un «mercantile State» (Harriss, 1984).

Les élus locaux, députés à l'assemblée législative de l’État ou plus souvent conseillers municipaux (corporators), représentent d'autres acteurs qui peuvent être tout à la fois contactés par les habitants pour obtenir des privilèges ou le respect de leurs droits en matière alimentaire, et parties prenantes dans la protection d'un agent ou dans la nomination d'un gérant de ration shop, voire dans certains cas de la mutation d'un fonctionnaire du PDS. Ils agissent moins en tant que représentants élus que comme notables - ce sont même parfois de véritables caïds (élire un criminel n’effraie pas les électeurs, du moment qu'il est puissant et donc protecteur et redistributeur). On pourrait dire que c'est le clientélisme plus que le souci de représentation démocratique d'une circonscription qui les fait intervenir, mais vu la polarisation ethnique des votes (caste, religion, etc.) prévalant en Inde, il n'est pas toujours facile de faire la part des choses?.

La structuration communautaire est renforcée par le poids des associations locales dans la gouvernance de quartier. Ces associations (community-based organisations) ont de plus en plus d'importance vu les politiques de développement participatif. Mais leur identité est moins spatiale qu’ethnique, et les «clubs de jeunes » ou les «associations de femmes » représentent souvent moins le quartier qu'une caste ou une confession. Mieux, ces associations sont souvent sous le parapluie protecteur d'un parti politique.

Association-parti-élu-agent-fonctionnaires, tel est donc le quintette avec lequel est en relation l'usager. Mais ce dernier n'est pas un individu ou un ménage isolé face à des institutions. Il est lui-même inséré dans des réseaux qui recoupent parfois ces institutions, et qui composent son «capital social».

9 Les discriminations et clivages au sein des «communautés» nuancent fortement bien des situations, et des groupes à l'intérieur de la communauté «favorisée» se plaignent souvent de n'être pas convenablement représentés par leur corporator. 


\section{CAPITAL OU CAMISOLE SOCIALE?}

\section{I. LA VERTICALITÉ DU CAPITAL SOCIAL}

Le «capital social» tel qu'il est défini par Putnam (2000) ou Woolcock (1998) et popularisé par le World Development Report (2004) recèle trois composantes. Le plus fermé est le capital communautaire (bonding social capital), entre pairs (famille, amis, etc.). Ce capital peut aussi être fondé sur la caste - même si le World Development Report ignore tout aspect ethnique pour n'évoquer que «voisins» ou «famille», etc. -, sur des groupes linguistiques, confessionnels, ou de classe. Dans nos enquêtes, il est matérialisé par la segmentation résidentielle (mon voisin est en général de la même communauté que moi) et par les associations locales.

Le capital-passerelle (bridging social capital) permet de créer des liens entre communautés: ce sont les associations professionnelles unissant hindous et musulmans, les pèlerinages fréquentés par plusieurs confessions, etc. Il est peu présent dans notre Inde populaire, ce qui se traduit par des tensions intercommunautaires quasi permanentes. Quant au capital de «relations» - au sens de Bourdieu (linking social capital) -, il relie verticalement à des personnes et institutions d'influence. Avoir de l'entregent est essentiel dans une société si hiérarchisée. Ces liens traversent les structures horizontales de classe, mais reprennent couramment les structures de caste ou de communauté linguistique ou religieuse, lesquelles sont souvent soutenues par un parti politique (Widmalm, 2008).

Dans notre étude comme en Inde en général, la conjonction du capital communautaire et du capital relationnel tend à associer une communauté à un protecteur, assurant une certaine sécurité mais renforçant le clientélisme voire la corruption, ce que nous appelons la «gouvernance verticale» (Landy et al., 20I3). Le capital communautaire qui selon la théorie de Putnam est la base de la démocratie fonctionne en Inde aussi comme un mode d'exploitation, puisqu'il est à la base de la dépendance envers le quintette décrit plus haut. Même s'il existe en réalité bien des exceptions à cette règle ${ }^{10}$, même

I0 II ne faudrait pas croire que la segmentation décrite suit parfaitement des lignes ethniques. Un même parti politique peut soutenir des associations de castes différentes, accueillir des militants d'origines régionales assez variées, et deux frères d'une même famille peuvent 
si les populations pauvres ne doivent pas être considérées comme de purs «agents/bénéficiaires passifs» (Benjamin et Bhuvaneswari, 2006, p. 228) du fait d'un certain pouvoir de négociation, il est fort difficile à un musulman des bidonvilles étudiés à Bombay de ne pas voter pour le parti du Congrès ou pour le parti cousin NCP. Son association sera sous la protection de ce parti (le président de l'association étant lui-même souvent membre du parti, à la croisée entre capitaux communautaire et relationnel). Et ce même parti tiendra un ou deux agents auprès du bureau local du PDS, où il aura peut-être obtenu contre rétribution la nomination du Rationing Officer. Le capital communautaire, avec derrière lui le ou les partis politiques, est un système de sécurité sociale, mais dont les «cotisations » se révèlent fort élevées pour les ménages.

\section{2. ÉTAT, SOCIÉTÉ CIVILE ET SOCIÉTÉ POLITIQUE}

En définitive, la corruption apparaît comme fortement institutionnalisée : ses circuits s'appuient sur les structures prévalentes de gouvernance tout en les renforçant, qu'elles soient administratives (relations hiérarchiques) ou sociales - ce que Harriss-White (2003) appelle «l'État de l'ombre» (shadow State). Certes, cela peut être perturbé par l'arrivée d'un fonctionnaire vertueux ou la mutation d'un corrompu. Mais d'une façon générale, les partis politiques ont besoin d’hommes forts localement qui leur gardent une «banque de voix» (Véron et al., 2006), fussent-ils des corporators notoirement véreux. Réciproquement, ceux qui refusent la corruption sont souvent forcés in fine de se concilier des individus ou des structures corrompus - et la tentation est ensuite forte d'accepter de vendre leur silence ${ }^{11}$.

parfois appartenir à des partis ou des associations différentes. Un habitant va en fait souvent utiliser un agent différent de son voisin. De même, on peut penser que le capital communautaire, aussi prégnant soit-il, est trop faible pour supprimer la corruption du PDS: il manque pour cela une mobilisation à l'échelle de la ration shop ou du Rationing Office, qui parvienne à construire un capital communautaire digne de ce nom, avec une base plus spatiale qu'ethnique. Si c'était le cas, les services publics ne fonctionneraient pas seulement en fonction du capital communautaire, mais ils pourraient à l'inverse aider à produire un tel capital, socialement et spatialement (pensons par exemple à la défense en France des services de soins ou d'éducation menacés de fermeture, qui soude la population de la petite ville ou du quartier).

II Bien des militants écologistes ou social workers de Bombay, après des années de lutte, finissent par accepter contre pots-de-vin d'abandonner une poursuite ou une dénonciation, basculant ainsi à leur tour dans la corruption. 
Qu'il existe une telle porosité entre les sphères de l’État et de la société civile (Mooij, 1999; Benjamin, Bhuvaneswari, 2006) fait qu'on peut difficilement maintenir qu'existe cette dernière en tant que telle. Partha Chatterjee est un des chercheurs indiens qui a le mieux contesté cette notion en la dédoublant par ce qu’il appelle la «société politique». Alors que la «société civile» est fondée sur l'égalité et l'autonomie individuelle, correspondant à un idéal finalement très occidental, ce que Chatterjee appelle la «société politique» est largement dominante notamment chez les classes sociales pauvres: elle est le domaine des médiateurs entre l'État et la population, des partis politiques notamment, souvent via du clientélisme. Bien des mobilisations de cette société politique se font «en violation de la loi» (Chatterjee, 200I, p. I77), comme par exemple la défense des bidonvilles.

L’État démocratique compose tant bien que mal avec elle. Politiciens et petits bureaucrates jouent un rôle essentiel dans le fonctionnement du everyday state (Véron et al., 2003). Mais, alors que Chatterjee voit un rôle parfois positif à cette «société politique», pour protéger les minorités ou les victimes des politiques de développement de l'État moderniste (grands barrages) ${ }^{12}$, une telle vision ne peut exister dans le cas de programmes comme le PDS qui sont destinés aux populations pauvres elles-mêmes: cette société politique ne peut que détourner, ou du moins biaiser, l'accès aux biens et services promis.

Pourtant, les habitants se satisfont que leurs associations locales soient à la fois communautarisées et très politisées. Cette structure pyramidale «habitants $<$ association < parti » ne leur apparaît pas écrasante, mais constitue au contraire une caisse de résonnance des plaintes et demandes, un moyen d'avoir un «capital social au carré » puisqu'une requête faite auprès de l'association est souvent transmise au parti. Les institutions et les personnes émanant de la démocratie indirecte ne sont pas considérées comme plus efficaces ni légitimes en soi que les notables ou que les partis politiques considérés dans leur aspect «réseau de relations». Ceci vaut pour les populations pauvres comme pour les populations

12 Une telle approche positive de l'importance de la «société politique» et du clientélisme est défendue à propos d'autres pays d'Asie, tels les succès économiques et le développement en Corée du Sud ou à Singapour qui sont en partie fondés sur les relations personnelles voire la corruption (Boesen, 2007). 
aisées dont les associations de quartier ou d'immeuble jouent un rôle crucial dans la gouvernance urbaine (Zérah, 2007). Il y a donc un grand pragmatisme dans le choix des personnes et institutions que les citadins contactent pour obtenir solutions ou privilèges. En outre, tout le monde a conscience que la séparation entre société civile et société politique est tout sauf imperméable: la présidente d'une association locale de femmes rencontrée à Bombay est aussi une politicienne, et elle travaille au ministère du Logement... Tout se passe comme si la société civile avait été «contaminée» par la société politique : peu d’associations des classes moyennes «modernes» ne sont pas basées sur une identité communautaire ou politique, sur une Gemeinschaft au moins autant que sur la Gesellschaft.

Il reste que les institutions d'intermédiation et de gouvernance créées par l'État, appelées «espaces invités » par Cornwall (2004), sont davantage utilisées par la société civile, par les populations diplômées et aisées (exception de taille: les élections, puisqu'en Inde la participation électorale est inversement proportionnelle au revenu). Les «espaces inventés » par la population (Miraftab, 2004), quant à eux, sont davantage le propre de la société politique (Harriss, 2007).

Tableau I. Espaces inventés et invités pour la gestion du PDS

\begin{tabular}{|l|l|l|}
\hline & \multicolumn{1}{|c|}{ Espaces invités (rares) } & \multicolumn{1}{|c|}{ Espaces inventés (dominants) } \\
\hline $\begin{array}{l}\text { Prise de parole (voice) } \\
\text { individuelle }\end{array}$ & $\begin{array}{l}\text { Boîte ou registre de plaintes à la } \\
\text { ration shop (souvent inexistant). }\end{array}$ & $\begin{array}{l}\text { Médiation d'un notable ou d'une } \\
\text { connaissance, clientélisme. }\end{array}$ \\
\hline Prise de parole collective & $\begin{array}{l}\text { Comité de vigilance (souvent non } \\
\text { fonctionnel). Elections locales. }\end{array}$ & $\begin{array}{l}\text { Manifestation, rasta roko (rue barrée), } \\
\text { gherao (siège du bureau des fonc- } \\
\text { tionnaires). }\end{array}$ \\
\hline
\end{tabular}

Une autre différence est que la société civile tend à exprimer ses requêtes en termes de droits à respecter, avec un caractère universaliste, tandis que la société politique les exprime plutôt en termes de privilèges dévolus à une communauté en particulier. Cela correspond à la distinction entre la corruption qui permet juste de faire valoir ses droits (livret de la bonne couleur) grâce à de la speed money, et celle qui permet d'avoir accès à des biens et services inaccessibles voire illégaux (permis de construire). Les perceptions de ces deux types de corruption seront évidemment assez différentes, de même qu’on ne considè- 
rera pas de la même façon un bakchich «honnête », de montant limité, et un pot-de-vin ruineux ou n’ayant pas donné accès au service escompté.

Toutes, cependant, peuvent donner des indications sur les représentations populaires de l'État. En Afrique (Olivier de Sardan, 1999) comme en Inde (Gupta, 1995), la stigmatisation de la corruption est générale. S’il est vrai qu’il y a peu de dénonciations effectives dans les actes, ce ne sont pas seulement les discours des membres de la «société civile», éduqués et «occidentalisés» qui portent cette critique, mais aussi ceux de la «société politique». On a donc là un paradoxe. D'un côté, la corruption fait partie du quotidien et du système de gouvernance local - à tel point qu'Olivier de Sardan parle d'«économie morale de la corruption» sans avoir peur de l'oxymore. De fait, les valeurs qui poussent à parler de «corruption» sont extrêmement subjectives : un fonctionnaire qui n'accorde pas de privilège à sa famille ou à son voisinage sera considéré comme égoïste par son entourage, mais s'il le fait il sera accusé de népotisme et de corruption par les autres. Non seulement légalité ne rime pas avec légitimité, mais encore la légitimité des uns est abus pour les autres. Le paradoxe est cependant que d'un autre côté, la dénonciation de la corruption est une manière de définir l'État en négatif, de «le constituer par son discours » (Gupta, 1995, p. 376). Critiquer les fonctionnaires, c'est par là même évoquer en creux les normes d'un État «moderne» quasi wébérien, c'est se considérer comme un «citoyen» avec des droits: une étape de plus donc dans la construction de l'État postcolonial et surtout de son adoption par une société véritablement $\left\langle\right.$ civile $»^{13}$. Voilà bien une évolution de longue haleine puisque l'État démocratique indien a été créé par Nehru et ses camarades alors qu'était embryonnaire la société civile. L'inverse avait eu lieu en Europe, où c'est la société civile qui a créé la démocratie (Véron et al., 2003).

13 Comme le souligne Gupta (1995, 2012), cet État est assurément multiforme, se situant en plusieurs lieux, en plusieurs administrations, à plusieurs échelles: le chercheur ne doit pas réifier un prétendu État qui n'existe pas dans les discours populaires. 


\section{MODERNITÉ ET STRUCTURES COUTUMIÈRES}

Rares sont les travaux cherchant à expliquer le pourquoi (et non point seulement le fonctionnement) de la petite corruption (Goetz, Jenkins, 2005). Olivier de Sardan (1999) l'a tenté pour l'Afrique occidentale : nous nous proposons, pour terminer, d'examiner ses hypothèses à l'aune du cas indien. Cette comparaison internationale pourra servir à monter en généralité, en expurgeant les facteurs propres à un continent ou à un autre.

Olivier de Sardan évoque en particulier six «logiques » sociales qui contribuent à la corruption africaine.

1. La «logique de négociation» est présente en Afrique dans les marchandages lors d'un achat, pour la conclusion d'un mariage entre deux familles, mais aussi pour les «arrangements ». Cette logique est aussi prégnante en Inde, et se manifeste dans la gouvernance du PDS. Dans les deux continents, ce souci de la négociation favorise l'existence d’intermédiaires.

2. Domine alors la «logique du courtage », que l'on retrouve par exemple avec les agents du PDS.

3. La tradition du «cadeau», qu'il faut donner à tout moment dans la vie quotidienne en Afrique, est présente en Inde quoique sans doute à un degré moindre. On pourrait la rapprocher d'une conception «non occidentale» des rapports à l'argent. Ainsi, Jonathan Parry (cité par Vidal, 2004) avait trouvé que chez les prêtres de Bénarès n'existait pas de distinction morale bien claire entre les dons et les transactions monétaires: les transactions en argent ou les relations de crédit, même inégalitaires, sont vues de façon plus neutre qu’en Occident, parce qu’en Inde il n’y a jamais eu d'idéal d’autarcie économique.

4. La «logique du réseau de solidarité »: ces réseaux sont très nombreux, depuis la famille (étendue) jusqu'à l'ethnie ou au parti politique, et ils impliquent mutuelle assistance. Dès lors, les transactions commerciales se font souvent à travers des réseaux de connaissances (Vidal, 2004, pour l'Inde) : soit directement entre l'acheteur et le vendeur (cas du commerce de détail), soit indi- 
rectement via des intermédiaires et courtiers, notamment pour le marché de gros très demandeur de crédit, et parce que dans tous les cas des relations de confiance traversent ces transactions. De telles pratiques se retrouvent dans l'accès aux services publics. Pour Olivier de Sardan, ce sont plutôt les gens manquant de ces réseaux qui doivent payer un pot-de-vin, pour compenser cette faiblesse. À Hyderabad ou Bombay, les ménages pauvres paient plus que les autres. Mais d'un autre côté, on pourrait affirmer que beaucoup de capital social favorise la corruption, dans la mesure où il permet de protéger des courtiers et toutes sortes de péages informels sous le parapluie du parti ou de l'association. Cette densité des réseaux, et du même coup cette faiblesse de l'individuation et de l'anonymat, qui est le propre de toute «face to face society» (Olivier de Sardan), expliquent le peu de dénonciations : l'opprobre jeté sur le plaignant serait trop grand, avec un risque de se couper de ses réseaux et d’une certaine sécurité sociale.

5. La «logique de l'accumulation redistributrice» explique qu'en Inde comme en Afrique un fonctionnaire qui refuse de s'enrichir sera considéré comme stupide mais aussi comme égoïste, étant donné la pression sociale pour une redistribution des richesses en faveur de la famille ou du réseau de clientèle. Jean-François Bayard (2006) a bien décrit cette combinaison d'intérêts individuels et de pression collective qui engendre la corruption en Afrique (voir aussi Eisenstadt, Roniger, 1984).

6. La «logique de la tradition prédatrice» est en revanche un facteur assez spécifique à l'Afrique : les abus de la colonisation puis de la décolonisation sont bien moins visibles en Inde, où l'État actuel est né d'une lutte pour l'Indépendance extrêmement dure, personnifié par deux hommes aussi emblématiques qu'intègres: Nehru et Gandhi. On peut donc penser, puisque la corruption est aussi généralisée dans les deux continents, que les facteurs historiques ne doivent pas être mis au premier plan pour l'Afrique.

Plus essentiel apparaît un processus commun à la plupart des pays du Sud : l'apparition d'une structure binaire, voire plurielle, que l'on retrouve dans de nombreux domaines, ceux du droit, de l'administration, et des pouvoirs en général. En Afrique, relève ainsi Olivier de Sardan (et dans beaucoup de cas 
en Inde), la tradition d'un «domaine public » est absente : au village, le foncier relève d'un chef de terre, et c'est souvent une autorité coutumière qui gère ce qui n'est «public » qu’en apparence. De même, il existe un pluralisme juridique caractérisé par la survivance des règles dites «traditionnelles», par la pluralité des droits musulman, hindou, «moderne», etc., sans parler des pouvoirs coutumiers qui coexistent avec les pouvoirs officiels. La coexistence de ces normes différentes est en soi facteur de corruption, ce dont témoigne l'existence des intermédiaires : ceux-ci sont capables d'un «syncrétisme historique » (Olivier de Sardan, 1999, p. 38) établissant des ponts entre les pratiques précoloniales, les héritages coloniaux et les transformations postcoloniales.

Il nous semble en définitive que la corruption est la conséquence d'un «plaquage» de valeurs et d'institutions, fondées sur l'individualisme et l'égalité - qui commencèrent à prédominer en Europe au XIX ${ }^{\mathrm{e}}$ siècle - sur des structures sociales où la collectivité joue un rôle encore majeur aujourd'hui, pour des raisons notamment de sécurité sociale, et où la notion d'égalité est encore moins diffusée en Inde qu'en Afrique : Louis Dumont (1966) n’y a-t-il pas écrit Homo Hierarchicus? Le processus d'ouverture socio-politique de la démocratie indienne, qui comme toute démocratie contemporaine se caractérise par une «dés-institutionnalisation » de certaines structures préexistantes et une «privatisation de la confiance», se superpose à une société encore «hiérarchisée et ascriptive » fondée sur des statuts prédéterminés dépassant les individus (Eisenstadt, Roniger, 2004, p. 297). Le contact entre les deux ne peut qu'engendrer du «jeu», facteur de corruption. Tout comme la sphère économique n'est pas isolée de la sphère socio-culturelle mais bien «enchâssée » dedans (voir Polanyi, 200I [1944]; ou Granovetter, 1985), de même l'administration ne se trouve pas dans une «tour d'ivoire» mais se trouve très perméable aux structures et logiques de la société dans laquelle elle fonctionne. C'est ce que Bayard (2006, p. I08) appelle l'«économie informelle de l'État » - un oxymore de plus, pour un thème qui décidément s'y prête... D’où le caractère «schizophrénique» du fonctionnaire (Olivier de Sardan, 1999), écartelé entre la logique bureaucratique officielle et les impératifs sociaux (sans que cela soit forcément mal vécu d’ailleurs). 
Tout se passe comme si la «modernité » avait été projetée sur des systèmes que l'on qualifiera par facilité de «coutumiers»: le cadeau s'est transformé alors en pot-de-vin, la solidarité de groupe est devenu népotisme. La corruption naît de la projection du moderne sur le coutumier. Assurément, une telle affirmation aussi schématiquement exposée pourra prêter à des critiques. Elle accepte en tout cas bien des nuances. Ainsi, alors qu'Olivier de Sardan évoque avant tout dans son argumentation les «villages» et les institutions précoloniales qui ont survécu (lignages, etc.), il ne faudrait pas penser que les structures coutumières évoquées sont forcément «traditionnelles » : le clan, le lignage, la caste, les solidarités confessionnelles ou régionales se sont fort bien adaptés aux structures formelles de la modernité, que ces dernières aient été créées par l'État postcolonial ou dérivées de son fonctionnement (partis politiques), ou qu'elles soient issues des nouveaux modes de «bonne gouvernance » (institutions de la décentralisation, associations locales, ONG, etc.). Gardons-nous d'une approche trop culturaliste voyant partout du «traditionnel» sans aucune historisation (Olivier de Sardan, 20ıо). «Le clientélisme n’est donc pas l'envers de la modernité » (Briquet, Sawicki, I998, p. 4). Mieux : la mondialisation, le brassage des hommes et des cultures à l'échelle planétaire mais aussi à l'intérieur même des pays, tendent dans bien des cas à accentuer paradoxalement les clivages et les identités, qu'elles soient confessionnelles, régionales, linguistiques, etc. L’Inde urbaine en est un bon exemple. Les structures «traditionnelles» sont en fait des structures très modernes - on pourrait dire plus exactement «post-modernes » - qui non seulement ont survécu aux profondes transformations des dernières décennies, mais ont pu se renforcer en prenant de nouveaux visages. 


\section{CONCLUSION}

À travers l'étude du PDS, l'identité des consommateurs apparaît hésiter entre cinq perspectives :

- Ils tentent d'être les usagers d'un service public, non sans difficulté;

- mais les réformes pour faire fonctionner davantage le PDS à prix coûtants tendent à les considérer comme des clients;

- clients, ils le sont aussi au sens du clientélisme, dont ils dépendent souvent ;

- dès lors, ils peinent à être considérés comme des citoyens, au sens à la fois de la nationalité (le livret sert de carte d'identité indienne) et de la démocratie (l'individu avec ses droits est moins reconnu que le groupe);

- certains, les habitants des bidonvilles les plus précaires, ne sont même pas reconnus comme des citadins.

Moins que jamais, le degré de corruption d'un pays apparaît avoir de relation avec son système de gouvernance. Assurément, plus une société locale est hiérarchisée, analphabète, soumise au clientélisme, et plus la corruption y sera facile (Véron et al., 2003). Pourtant, l'Inde où les gouvernements se succèdent démocratiquement selon la règle de l'alternance, et ce à toutes les échelles, demeure $94^{\mathrm{e}}$ sur 176 au classement 2012 de Transparency International. Constat semblable quand on descend au niveau des États fédérés et des régions. Alors que la corruption était jadis attribuée au «tout-État» (Harriss-White, White, 1996), les processus de décentralisation et participation ne font souvent qu'aggraver les choses, engendrant une « décentralisation de la corruption» (Véron et al., 2006).

C'est là tout l'intérêt d'une analyse en termes de «société politique » ${ }^{14}$. Elle permet de comprendre que «participation» n’est pas synonyme de «démocratisation », la première pouvant même saper la seconde: à Delhi, de bourgeoises associations locales ou de résidents court-circuitent les élus (qui sont souvent des femmes ou de basse caste en raison de la discrimination positive) pour contacter directement des niveaux de pouvoir plus élevés afin d’obtenir des passe-droits (Mooij, Tawa Lama-Rewal, 2009). De même, l'exemple du PDS montre que les

14 Cette approche apparaît complémentaire d'une analyse plus centrée sur les logiques bureaucratiques et les cultures professionnelles pour expliquer la corruption (Bierschenk, Olivier de Sardan, 2014). 
institutions dites de la société civile et les partis politiques, loin de jouer le jeu d’une démocratie «idéale» telle quelle peut être imaginée en Occident, peuvent fonctionner au service de privilèges, de la segmentation sociale et de la corruption. Les associations locales peuvent protéger les populations pauvres en engendrant des contre-pouvoirs, mais aussi se servir de leur nombre pour fonctionner comme des «courroies de transmission » qui renforcent le pouvoir de notables sur les masses à travers différents intermédiaires tels que les agents du PDS. Loin d’introduire toujours plus de transparence, la nouvelle gouvernance que les bailleurs de fonds internationaux tentent d'instaurer n'est pas nécessairement si «bonne».

Les remèdes à la corruption, on le voit, n'existent que très en amont: le renforcement d'un système public et institutionnalisé de sécurité sociale et de retraite, en particulier, supprimerait bien des recours aux réseaux sociaux informels, et permettrait une avancée de la société civile face à la société politique.

\begin{abstract}
NDA. Merci au Centre de Sciences Humaines de New Delhi et à l'Institut Universitaire de France d'avoir collaboré à cette recherche, à toute l'équipe APUG, ainsi qu'aux étudiants et assistants T. François, C. Hachet, D. Ruby, P. Sekhsaria qui ont passé 16 mois sur le terrain - 8 semaines pour F. Landy. Des enquêtes-ménages avec questionnaires semi-ouverts ont été complétées par des entretiens avec des acteurs de tous ordres (habitants, fonctionnaires, élus, commerçants, etc.). Merci aussi à J.P. Olivier de Sardan pour ses remarques.
\end{abstract}

\title{
BIBLIOGRAPHIE
}

BAYARD J.F., 2006 (1989), L'État en Afrique. La politique du ventre, Paris, Fayard.

BENJAMIN S., BHUVANESWARI R., 2006, «Urban futures of poor groups

in Chennai and Bangalore», in Local Governance in India, N. Gopal Jayal,

A. Prakash, P.K. Sgarma (éds)., New Delhi, Oxford University Press, p. 221-267.

BERTRAND M., DJANKOV S., HANNA R., MULLAINATHAN S., 2008,

«Corruption in Driving Licensing Process in Delhi», Economic and Poilitical Weekly, 43-5, p.7I-76.

BIERSCHENK T., CHAUVEAU J.P., OLIVIER DE SARDAN J.P. (éds), 2000, Courtiers en développement. Les villages africains en quête de projets, Paris, Karthala. 
BIERSCHENK T., OLIVIER DE SARDAN J.P. (éds), 2014, State at works. The Dynamics of African Bureaucracies, Leiden, Brill.

BLUNDO G., OLIVIER DE SARDAN J.P. (éds), 2007, L'État et la corruption en Afrique, Paris, Karthala.

BOESEN N., 2007, « Governance and Accountability: How Do the Formal and Informal Interplay and Change? », in Informal Institutions: How Social Norms Help or Hinder Development, J. Jütting et al. (éds), Paris, OCDE, p. 83-100.

BRIQUET J.L., SAWIKI F. (éds), 1998, Le Clientélisme politique dans les sociétés contemporaines, Paris, PUF.

CHATTERJEE P., 200I, «On civil and political society in postcolonial democracies», in Civil Society. Histories and Possibilities, S. Kaviraj, S. Khilnani (éds), Cambridge, Cambridge University Press.

CORNWALL A., 2004, «Introduction: New Democratic Spaces? The Politics and Dynamics of Institutionalised Participation », IDS Bulletin, 35-2, p. I-10.

DAS S.K., 200I, Public Office, Private Interest: Bureaucracy and Corruption in India, New Delhi, Oxford University Press, 2001.

DUMONT L., 1966, Homo Hierachicus. Essai sur le système des castes, Paris, Gallimard.

EISENSTADT S.N., RONIGER L., 1984, Patrons, Clients and Friends. Interpersonal Relations and the Structure of Trust in Society, Oxford, Oxford University Press.

GRANOVETTER M., 1985, «Economic Action and Social Structure: The Problem of Embeddedness », The American Journal of Sociology, 91-3, p. 481-510.

GOETZ A-M., 2007, «Political Cleaners: Women as the New Anti-Corruption Force?», Development and Change, 38-I, p.87-105.

-, JENKINS R., 2005, Reinventing Accountability: Making Democracy Work for Human Development, New York, Palgrave-MacMillan.

GUÉRIN I., KUMAR S., 2007, «Clientélisme, courtage et gestion des risques en microfinance. Étude de cas en Inde du Sud», Autrepart, 4-44, p. 13-26.

GUPTA A., 1995, «Blurred boundaries: the discourse of corruption, the culture of politics and the imagined state», American Ethnologist, 22, p. 375-402. 
-, 2012, Red Tape. Bureaucracy, Structural Violence and Poverty in India, Durham, Duke University Press.

HARRISS B., 1984, State and Market. State Intervention in Agricultural Exchange in a Dry Region of Tamil Nadu, New Delhi, Concept.

HARRISS-WHITE B., 2003, India Working. Essays on Society and Economy, Port Chester (New York), Cambridge University Press.

-, WHITE G., 1996, «Corruption, Liberalization and Democracy: Editorial Introduction», IDS Bulletin, 27-2, p. I-5.

HARRISS J., 2007, «Antinomies of Empowerment. Observations on Civil Society, Politics and Urban Governance in India», Economic and Political Weekly, 42-26, p. 2716-2724.

KUMAR G., LANDY F., 2009, «Vertical governance: Brokerage, patronage and corruption in Indian metropolises», in Ruet, Tawa Lama-Rewal (2009), p. I05-I34.

LANDY F., 2006, Un milliard à nourrir. Grain, territoire et politiques en Inde, Paris, Belin. -, FRANCOIS T., RUBY D., SEKHSARIA P., 2013, «Gouvernance verticale et corruption en Inde urbaine: la segmentation spatiale de l'aide alimentaire publique», L'Espace Politique, 21-3:<http://espacepolitique.revues.org/28|2>.

LEVINE R.M., 1997, Brazilian Legacies, New York, M.E. Sharpe.

MIRAFTAB F., 2004, «Invented and Invited Spaces of Participation», Wagadu. Journal of Transnational Women's and Gender's Studies, I : <http://web.cortland. edu/wagadu>.

MOOIJ J., 1999, Food Policy and the Indian State, New Delhi, Oxford University Press.

-, TAWA LAMA-REWAL S., 2009, «Class in Metropolitan India: The Rise of the Middle Classes», in Ruet, Tawa Lama-Rewal (2009), p. 81-104.

OLIVIER DE SARDAN J.P., 1999, «A Moral Economy of Corruption in Africa?», The Journal of Modern African Studies, 37-I, p. 25-52.

-, 200I, «La sage-femme et le douanier. Cultures professionnelles locales et culture bureaucratique privatisée en Afrique de l'Ouest», Autrepart, 20, p.61-73.

-, 2010, «Le culturalisme traditionaliste africaniste. Analyse d'une idéologie scientifique», Cahiers d'Études africaines, 198-199-200, p.419-453. 
-, 2014, «The Delivery State in Africa. Interface Bureaucrats, Professional Cultures and the Bureaucratic Mode of Governance», in Bierschenk, Olivier de Sardan (2014), p399-429.

POLANYI K., 200I (1944), The Great Transformation. The Political and Economic Origins of Our Time, Boston, Beacon Press.

PUTNAM R., 2000, Bowling Alone: The Collapse and Revival of American Community, New York, Simon \& Schuster.

ROSENN K. S., 197I, «The Jeito. Brazil's Institutional Bypass of the Formal

Legal System and its Development Implications», The American Journal of Comparative Law, 3, p. 514-549.

RUET J., TAWA LAMA-REWAL S. (éds), 2009, Governing India's Metropolises, New Delhi, Routledge.

SHROFF M.F., 2008, Breathless in Bombay, New Delhi, Picador.

SRINIVAS M.N., 1976, The Remembered Village, Berkeley, University of California Press.

VÉRON R., CORBRIDGE S., WILLIAMS G., SRIVASTAVA M., 2003, «The Everyday

State and Political Society in Eastern India: Structuring Access to the Employment

Assurance Scheme», The Journal of Development Studies, 39-5, p. I-28.

-, 2006, «Decentralized Corruption or Corrupt Decentralization?

Community Monitoring of Poverty-Alleviation Schemes in Eastern India», World Development, 34-II, p. 1922-1941.

VIDAL D., 2004, «Markets», in V. Das, Handbook of Indian Sociology, New Delhi, Oxford University Press, p. 388-40I.

WADE, R. 1985, «The Market for Public Office: Why the Indian State is not Better at Development», World Development, 13-4, p. 476-497.

WIDMALM S., 2008, Decentralisation, Corruption and Social Capital. From India to the West, New Delhi, Sage.

WOOLCOCK, M., 1998, «Social capital and economic development. Toward a theoretical synthesis and policy framework», Theory and Society, 27, p. 151-208.

WORLD BANK, 2004, World Development Report 2004: Making Services Work for Poor People, Washington. 
